\title{
Performance And Cellular Capacity Of $M$-ary PSK In Co-channel Interference
}

\author{
Hans Roelofs, Jeroen Thijs, and Rajan Srimivasan \\ Telecommunication Engineering Group, University of Twente \\ P.O. Box 217, 7500 AE Enschede, The Netherlands \\ Tel. +31 0534892108 Fax. +31 0534895640 \\ h.roelofs@ieee.org
}

\begin{abstract}
Fast simulation techniques are applied to estimate error rates of coherent $M$-ary phase shift keying (PSK) in the presence of co-channel interference $(\mathrm{CCI})$ and additive white Gaussian noise (AWGN). This type of interference occurs often in wireless systems that employ frequency reuse. Error rates are frequently calculated assuming that $\mathrm{CCI}$ can be modelled as Gaussian. It is shown that this assumption is valid only in noise dominated environments. Simulation techniques based on adaptive importance sampling (IS) are developed for this problem. Several new numerical results are presented and used to calculate cellular capacities.

Keywords Importance Sampling, Co-channel interference, $M$-ary PSK, Cellular capacity.
\end{abstract}

\section{INTRODUCTION}

Many digital communication systems are perturbed by interference that can be modelled as a sum of sinusoids with random phases. Mobile wireless systems often operate in interference dominated environments which can have a limiting effect on performance in terms of bit error rates and cellular capacities. In particular, $\mathrm{CCI}$ in such systems arises from frequency reuse in certain fixed patterns of geographic cells, [1]-[2]. The information bearing signal in a particular frequency cell is interfered by signals arriving from surrounding cells that use the same frequency. These randomly phased interfering signals add up, giving rise to $\mathrm{CCI}$.

Performance analysis in terms of bit or symbol error rates is usually carried out by making the simplifying assumption that $\mathrm{CCI}$ can be considered Gaussian. This yields accurate results only when the signal to (additive white Gaussian) noise ratios are low compared to the corresponding signal to interference ratios. Hence more accurate methods are required in interference dominated situations wherein the combination of Gaussian noise and interference is decidedly non- Gaussian. One fast simulation method, based on adaptive IS [3] - [5], is developed here for the coherent detection of $M$-ary PSK signalling. It is known that properly designed IS simulations can provide re- markable gains over Monte Carlo (MC) procedures in terms of computational effort for the estimation of rare event probabilities. In the following sections we adopt a simple structure for $\mathrm{CCI}$, develop biasing methods for phases of interferers and noise, describe implementation of adaptive estimators, present symbol error rates, and calculate cellular capacity for $M$ - ary PSK. Analysis is carried out for interference dominated situations as well as those consisting of interference and additive white Gaussian noise (AWGN) in nonfading channels. Binary and $M$-ary PSK are treated separately.

\section{CO-CHANNEL INTERFERENCE}

The following assumptions are made on the interfering signals:

- The carrier signal amplitude of the desired information bit stream is $A$ and that of the interfering signals is $\alpha A$ where $\alpha$ is a positive constant. The $L$ interferers are assumed to have equal amplitudes.

- Interfering signals are assumed to be similarly modulated as the desired signal but carry different equally likely information bits.

- The $i$-th interfering signal differs in phase from the desired signal by $\phi_{i}$. The set $\left\{\phi_{i}\right\}_{1}^{L}$ consists of random independent phases, uniformly distributed in $(0,2 \pi)$. The interfering carriers are at the same frequency as the desired signal. It is assumed that interfering signals are bit synchronized with the desired signal, resulting in all the energy of the interferer appearing at the demodulator output. This is a worst-mase situation.

\section{BINARY PSK}

The optimum receiver for coherent IPSK is a correlation detector or a matched filter-sampler followed by a zero threshold decision. It is assumed that a +1 information bit is transmitted, corresponding to a zero phase offset. The decision statistic at the demodulator output with $L$ interferers is proportional to

$$
A+n+\sum_{i=1}^{L} \alpha A \cos \phi_{i}
$$


where $\boldsymbol{n}$ is Gaussian and has zero mean with variance $\sigma_{n}^{2}$. The error probability can be written as

$$
\begin{aligned}
P_{e} & =P\left(A+n+\sum_{i=1}^{L} \alpha A \cos \phi_{i} \leq 0\right) \\
& =E\left\{1\left(A+n+\sum_{i=1}^{L} \alpha A \cos \phi_{i} \leq 0\right)\right\}
\end{aligned}
$$

where the indicator function $1(\cdot)=1$ if the event in its argument occurs and is zero otherwise. Each cosine term in the above has the probability density function shown as the solid line in Figure 1. Calculating the density of their sum is a computationally intensive task, involving an $L$-fold convolution. Assuming that the sum can be characterized by an equivalent Gaussian density (based on the central limit theorem) leads to

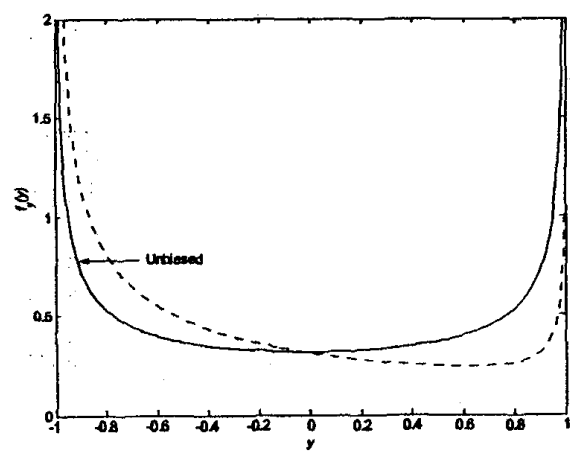

Figure 1: Pdf of a random phàse interfering signal

inaccurate answers, as shown later. While there exists an analytical approximation for the sum density [6], it is advantageous to resort to IS simulation to estimate $P_{e}$. This method is general and is applicable to a broad class of estimation problems. The IS estimator $\widehat{P}_{e}$ of $P_{\mathrm{e}}$ is given by

$$
\begin{aligned}
\widehat{P}_{e}= & \frac{1}{K} \sum_{k=1}^{K} 1\left(A+n+\sum_{i=1}^{L} \alpha A \cos \phi_{i} \leq 0\right) \\
& \cdot W\left(\phi_{1}, \ldots, \phi_{L}, n\right), \quad f \sim f_{\star}
\end{aligned}
$$

where the notation " $f \sim f_{*}$ " denotes that the $K$-length simulation is carried out with the original densities $(f)$ of the random variables replaced by biasing densities $\left(f_{\star}\right)$ that increase the frequency of occurrence of error events. The weighting function $W$ ensures unbiased estimates. Biasing densities are chosen such that the variances of the estimates are smaller than those obtained without any biasing for an $\mathrm{MC}$ simulation of equal length.

We consider biasing of interference phases and additive noise separately. The interference cosine terms need to have increased probability mass in the negative regions of the support of the density. An effective way to bias the phases $\phi_{i}$ is to increase the probability mass in the vicinity of $\phi_{i}=\pi$. We use a Gaussian biasing density with mean at $\pi$ and a common variance of $\sigma_{\phi}$. An example of the biased density for $\cos \phi$ is shown dashed in Figure 1 for $\sigma_{\phi}=1.5$. The Gaussian simulation samples that fall outside the $(0,2 \pi)$ interval are wasted, but the loss in efficiency is small.

For biasing the noise $n$, it is clear that endowing it with a negative mean would increase the probability of making detection errors. While variance scaling can be used, in this case it will not be as efficient as translating the mean. Denoting translation with parameter $c$, the weighting function is

$$
\begin{aligned}
& W\left(\phi_{1}, \ldots, \phi_{L}, n ; \sigma_{\phi}, c\right) \equiv \frac{f\left(\phi_{1}, \ldots, \phi_{L}, n\right)}{f_{+}\left(\phi_{1}, \ldots, \phi_{L}, n ; \sigma_{\phi}, c\right)}= \\
& \left\{\begin{array}{cc}
\left(\frac{\sigma_{\phi}}{\sqrt{2 \pi}}\right)^{L} \exp \left(\frac{\sum_{1}^{L}\left(\phi_{1}-\pi\right)^{2}}{2 \sigma_{\phi}^{2}}+\frac{c^{2}-2 c n}{2 \sigma_{n}^{2}}\right), \\
0 \leq \phi_{i} \leq 2 \pi \\
0, & \text { elsewhere }
\end{array}\right.
\end{aligned}
$$

To implement the estimator of (3.4), it remains to choose good values of the biasing parameters $\sigma_{\phi}$ and $c$. This is done in an adaptive 2-dimensional optimization that determines optimum values such that the variance of the estimator is minimized. This has been adequately described in [4] and [5]. They use stochastic Newton recursions that require the partial and mixed derivatives $\partial W / \partial \sigma_{\phi}, \partial W / \partial c, \partial^{2} W / \partial \sigma_{\phi}^{2}, \partial^{2} W / \partial c^{2}$, and $\partial^{2} W / \partial \sigma_{\phi} \partial c$. All these are easily obtained from (3.5). The IS optimization algorithm is the stochasic Newton recursion

$$
\left(\begin{array}{c}
\sigma_{\phi, m+1} \\
c_{m+1}
\end{array}\right)=\left(\begin{array}{c}
\sigma_{\phi, m} \\
c_{m}
\end{array}\right)-\delta \widehat{\boldsymbol{J}}_{m}^{-1} \cdot \widehat{\nabla} I\left(\sigma_{\phi, m}, c_{m}\right)
$$

where $\delta$ is the step size of the recursion and $\widehat{J}_{m}^{-1}$ is the inverse of the matrix $\widehat{J}_{m}$ given by

$$
\widehat{\boldsymbol{J}}_{m}=\left(\begin{array}{cc}
\hat{I}_{\sigma_{\phi, m} \sigma_{\phi, m}} & \hat{I}_{\sigma_{\phi, m} c_{m}} \\
\hat{I}_{\sigma_{\phi, m} c_{m}} & \cdot \hat{I}_{c_{m} c_{m}}
\end{array}\right)
$$

where $I_{x y} \triangleq \partial I_{x} / \partial y$. The estimated gradient operator $\widehat{\nabla}$ is

$$
\widehat{\nabla} I\left(\sigma_{\phi, m}, c_{m}\right)=\left(\begin{array}{ll}
\hat{I}_{\sigma_{\phi, m}} & \hat{I}_{c_{m}}
\end{array}\right)^{T}
$$

where $I_{\sigma_{\phi, m}}=\partial I\left(\sigma_{\phi, m}, c_{m}\right) / \partial \sigma_{\phi, m}$ and $I_{c_{m}}=$ $\partial I\left(\sigma_{\phi, m}, c_{m}\right) / \partial c_{m}$, and

$$
\begin{aligned}
& I\left(\sigma_{\phi, m}, c_{m}\right)= \\
& E\left\{1\left(A+n+\sum_{i=1}^{L} \alpha A \cos \phi_{i} \leq 0\right)\right. \\
& \left.. W\left(\phi_{1}, \ldots, \phi_{L}, n\right)\right\}
\end{aligned}
$$

is a quantity proportional to the estimator variance, the expectation being taken over the unbiased distributions. All terms with hats are estimates obtained during the IS 
simulation as part of the optimization algorithm. Simulations have been carried out for $L=6$ interferers for a wireless mobile communications network with hexadiagonal cell structure in which interfering cells use the same frequency as the cell under study. The 6 cells closest to the cell under study are at an equal distance while all other cells using the same frequency are at a larger distance and assumed to contribute negligible interference compared to the first tier.

The signal to interference ratio SIR is defined as $1 / L \alpha^{2}$, whereas the signal to noise ratio SNR is defined as $A^{2} / 2 \sigma_{n}^{2}$. The IS sample size is set to $K=10,000$ for all simulations. Results are shown in Figure 2 as a function of SIR with SNR as parameter For an SIR of $8.5 \mathrm{~dB}$ and SNR of $29 \mathrm{~dB}$, a simulation gain of $8.6 \times 10^{8}$ at an error rate of $2.4 \times 10^{-10}$ is obtained. A relative accuracy better than $6.6 \%$ for $P_{e}$ estimation is obtained for all cases. For finite SNR an error floor exists as the SIR becomes large.

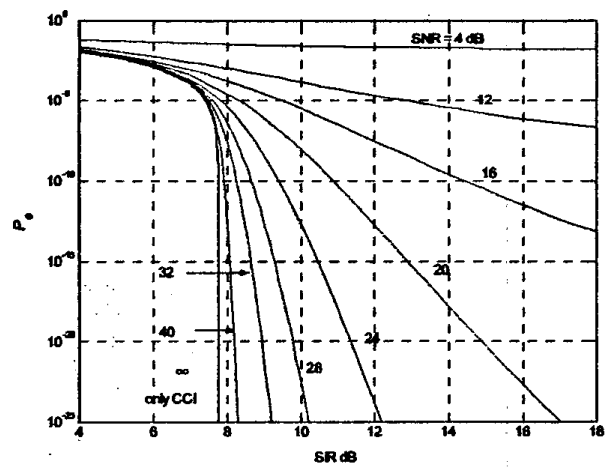

Figure 2: Error probabilities for BPSK with $\mathrm{CCI}$ and AWGN. Parameter is SNR

\subsection{Gaussian assumption}

The effect of modelling the CCI as Gaussian is examined by calculating the bit error rate assuming that the interference can be replaced by a Gaussian noise source having the same total power, in addition to ther$\mathrm{mal}$ noise of course. Defining a signal to interference and noise ratio SNIR as

$$
\mathrm{SNIR}=\frac{A^{2}}{L \alpha^{2} A^{2}+2 \sigma_{n}^{2}}
$$

the error rate is approximated as

$$
P_{\mathrm{e}} \approx Q(\sqrt{2 \mathrm{SNIR}})
$$

where $Q(x) \equiv \int_{x}^{\infty} e^{-y^{2} / 2} \mathrm{~d} y / \sqrt{2 \pi}$. This is shown in Figure 3, together with optimized IS estimates of $P_{e}$ for comparison. For low SNRs, that is in noise dominated situations, the Gaussian approximation is close to the IS estimates. As the SNR increases, in the interference dominated situation, the approximation becomes increasingly worse. This illustrates the importance of making accurate simulation estimates of performance in interference limited environments in preference to using Gaussian approximations.



Figure 3: Comparison of Gaussian approximations (GA) and IS simulation for BPSK.

\section{$4 \quad M$-ARY PSK}

Transmitting $M$-ary symbols using PSK, $\log _{2} M$ bits are encoded into each symbol in terms of the phases of the carrier. The optimum receiver is equivalent to a phase detector that computes the phase of the received signal vector and selects that symbol whose phase is closest. Assume that zero phase has been transmitted. From the signal space diagram of Figure 4, in which $L=1$, the phase $\phi_{r}$ of the received vector can be easily obtained. A correct detection is made when $\phi_{\text {r satisfies }}$ $-\pi / M \leq \phi_{r} \leq \pi / M$. Defining $\mathrm{I}(\cdot)=1-1(\cdot)$, the probability of a symbol exror can be written as

$$
\begin{aligned}
P_{\mathrm{e}} & =P\left(-\pi / M \leq \phi_{r} \leq \pi / M\right) \\
& =E\left\{\mathrm{~T}\left(-\pi / M \leq \phi_{r} \leq \pi / M\right)\right\}
\end{aligned}
$$

and its IS estimate as

$$
\begin{aligned}
\widehat{P_{e}}= & \frac{1}{K} \sum_{k=1}^{K} \mathrm{I}\left(-\pi / M \leq \phi_{r} \leq \pi / M\right) \\
& \cdot W\left(\phi_{1}, \ldots, \phi_{L}, n_{i}, n_{q}\right)
\end{aligned}
$$

where $n_{i}$ and $n_{q}$ denote the inpluase and quadrature noise components. The indicator for the complement of the event $\left\{-\pi / M \leq \phi_{r} \leq \pi / M\right\}$ can be simulated by referring to Figure 4 and noting that the error region comprises of the region $\left\{n_{i}+A+\alpha A \sum_{i=1}^{L} \cos \phi_{i} \leq\right.$ o\} together with (union) the intersection

$$
\begin{aligned}
& \left\{n_{i}+A+\alpha A \sum_{i=1}^{L} \cos \phi_{i}>0\right\} \\
& \bigcap\left\{\left\{\tan \phi_{r} \geq \tan (\pi / M)\right\}\right. \\
& \left.\cup\left\{\tan \phi_{r} \leq-\tan (\pi / M)\right\}\right\}
\end{aligned}
$$

We note that the distribution of the received phase $\phi_{r}$ is symmetric around $\phi_{r}=0$ by virtue of the fact that all the interference phases are independent and uniformly distributed, the same being true for the phase 


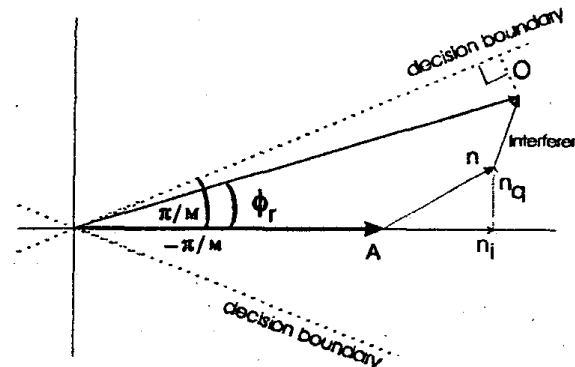

Figure 4: $M$-ary PSK signal space with a single cochannel interferer and AWGN

of the noise vector. Hence it is sufficient to simulate events from only (the upper) half of the error region described above. This can be described by considering the intersection of the complete error region and the set $\left\{\sin \phi_{r} \geq 0\right\}$.

Consequently, an effective method of biasing is to generate all interference phases $\phi_{i}$ such that vectors are most likely to be aligned along the line marked $O$ in Figure 4. This is evident from orthogonality and will produce an increase in the frequency of occurrence of errors. Hence the biased interfering phases are chosen as Gaussian with their means at $\mu \equiv(\pi / 2)+\pi / M$ and common variance $\sigma_{\phi}$, optimized through adaptive simulation. Increasing the variance of the noise $n$ will produce more errors. However this will lead to a more than 2-dimensional optimization problem. A simple solution is to translate the means of the quadrature noise variables along the shortest line to the decision boundary, as is done for the interferers. Denoting the biased means of the noise components as $c_{i}$ and $c_{q}$, it follows from Figure 4 that they should be related as

$$
c_{i}=-c_{q} \tan \frac{\pi}{M}
$$

This results in a 2-dimensional biasing problem involving $c_{i}$ (or $c_{q}$ ) and $\sigma_{\phi}$. The weighting function is

$$
\begin{aligned}
& W\left(\phi_{1}, \ldots, \phi_{L}, n_{i}, n_{q} ; \sigma_{\phi}, c_{q}\right)= \\
& \begin{cases}\left(\frac{\sigma_{\phi}}{\sqrt{2 \pi}}\right)^{L} \exp \left(\frac{\sum_{1}^{L}\left(\phi_{i}-\mu\right)^{2}}{2 \sigma_{\phi}^{2}}+\frac{\left(1+a^{2}\right) c_{q}^{2}+2 c_{q}\left(a n_{i}-n_{q}\right)}{2 \sigma_{n}^{2}}\right), \\
0 \leq \phi_{i} \leq 2 \pi \\
0, & \text { elsewhere }\end{cases}
\end{aligned}
$$

where $a \equiv \tan (\pi / M)$. The various derivatives can easily be obtained. Simulations are carried out in a similar manner to the binary PSK case. Results are shown in Figure 5. The SNR per bit is defined as $A^{2} /\left(2 \sigma_{n}^{2} \log _{2} M\right)$ and the SIR as $1 /\left(L \alpha^{2} \log _{2} M\right)$. The symbol error rate performances for QPSK differ only slightly from those for binary PSK. The same general remarks on error rate performance can be made as in the case of binary PSK.

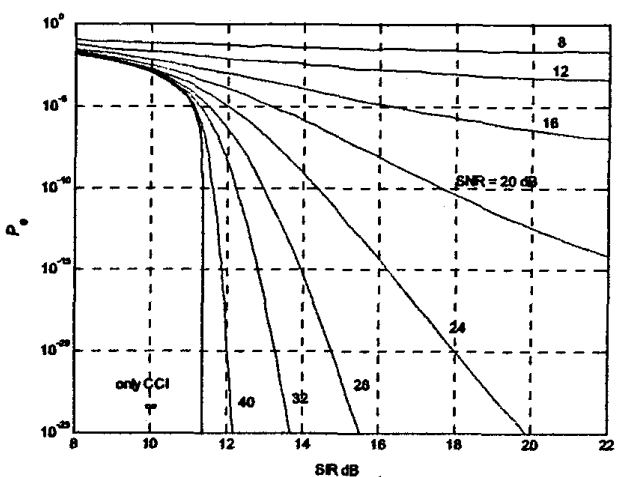

Figure 5: Error probabilities for 8-PSK with $\mathrm{CCI}$ and AWGN. Parameter is SNR

\subsection{Capacity}

Using error rate estimates, we can find the capacity per cell for $M$-ary PSK. To calculate this capacity as a function of the SNR, SIR and error rate, more parameters need to be defined. The required bandwidth is set to the null-to-null bandwidth. Hence, bandwidth efficiency $\eta$ for $M$-ary PSK is $\eta=\frac{1}{2} \log _{2} M$. In the hexadiagonal structure, the number of cells in a reuse pattern is denoted by the reuse factor $K_{\text {cell. }}$. The cell structure allows a set of values given by $K_{\text {cell }}=i^{2}+i j+j^{2}$, where $i$ and $j$ are two non-negative integers [2]. A common method to achieve performance better than some specified error rate is to increase the ratio $D / R$, where $D$ is the distance between centers of two cochannel cells and $R$ the cell radius. The $D / R$ ratio is related to SIR as

$$
\operatorname{SIR}=\frac{1}{6}\left(\frac{D}{R}\right)^{\gamma}
$$

where $\gamma$ is the path loss exponent. The relation between $D / R$ and $K_{\text {cell }}$ is found by noting that the perpendicular cell diameter is equal to $\sqrt{3} R$. This results in $D / R=\sqrt{3 K_{\text {cell }}}$. Substituting in (4.7), with $\gamma=4$ yields SIR $=\frac{3}{2} K_{\text {cell }}^{2}$. Finally, capacity per cell is defined as $C=\eta / K_{\text {cell. }}$. Substitution yields

$$
C=\frac{\frac{1}{2} \log _{2} M}{\sqrt{\frac{2}{3} \mathrm{SIR}}} \quad[\text { bits / s / Hz / cell }]
$$

To compare $M$-ary PSK schemes, it is assumed that symbols are equally likely and use Gray encoding. Hence, symbol error rate is well approximated by bit error rate. The following procedure is used to find the capacity per cell as a function of BER. For specified SNR and BER, the lowest SIR is found that satisfies the BER condition. Capacity per cell then follows from (4.8). Results are shown for 8-PSK in Figure 6. The capacity per cell is presented in two ways. First, as a continuous function of BER, ignoring the fact that $K_{\text {cell }}$ only takes non-negative integer values. Second, 


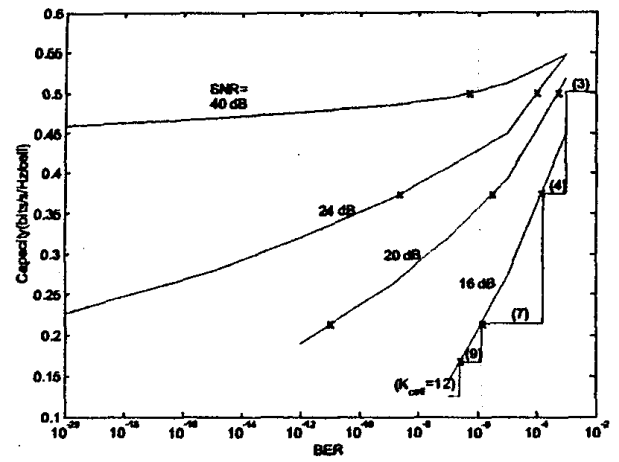

Figure 6: Capacity per cell as a function of the required bit error rate for 8-PSK. Markers indicate the transition of $K_{\text {cell. }} . K_{\text {cell }}$ values are in parenthesis. Parameter is SNR.

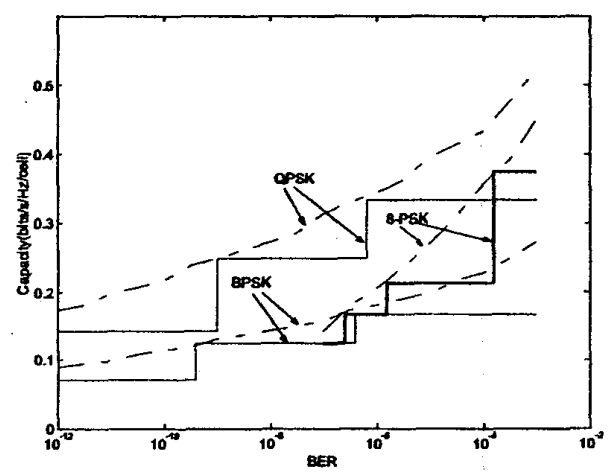

Figure 7: Comparison of capacity per cell for $\mathrm{SNR}=$ $16 \mathrm{~dB}$. Dashed lines represent the continuous capacity graphs. Solid lines represent the discrete capacity values.

as a discrete function of BER, taking into account that $K_{\text {cell }} \in\{1,3,4,7, \ldots\}$. For clarity, the latter is only shown for SNR $=16 \mathrm{~dB}$. Markers are placed on the continuous capacity graphs to indicate transitions of $K_{\text {cell }}$. Reuse factors are indicated at the discrete capacity values, between parenthesis.

For most combinations of BER and SNR, QPSK provides the maximum capacity per cell. This is illustrated more explicitly for SNR $=16 \mathrm{~dB}$ in Figure 7. Comparing discrete capacity values at SNR $=16 \mathrm{~dB}$, we conclude that QPSK provides the highest capacity per cell for $10^{-12} \leq \mathrm{BER} \leq 2 \cdot 10^{-4}$. QPSK is outperformed by 8-PSK in a small region $2 \cdot 10^{-4} \leq$ BER $\leq 10^{-3}$. For higher SNR, the region in which 8-PSK outperforms QPSK extends towards lower BER. The opposite occurs for lower SNR. Furthermore, BPSK is outperformed by QPSK for any combination of BER and SNR.

\section{CONCLUSION}

We have demonstrated how adaptive IS methods can be used to solve performance estimation problems that are analytically intractable. These would be computationally intensive if one were to resort to MC simulations. Although numerical results were displayed only for $M$-ary PSK, results for other signal sets can easily be obtained. In particular, it has been shown that the assumption of a Gaussian model in an interference dominated situation is not justified for evaluating error rates. The performance results are used to calculate the cellular capacity of wireless systems by incorporating propagation models and bandwidth considerations. It was found that QPSK outperforms BPSK and 8-PSK for most BER and noise levels. Further, the techniques developed here can be applied to different modulation schemes as also to channels characterized by fading and multipath. In [6] an interference dominated channel model was considered and Gaussian noise assumed absent. By including Gaussian noise in the channel model, more realistic performance analysis and capacity results have been obtained.

\section{References}

[1] Feher, K: Wireless digital communications, Prentice-Hall, New Jersey, 1995.

[2] Rappaport, T.S.: Wireless communications: Principles and Practices, Prentice-Hall, New Jersey, 1996.

[3] Remondo, D., Srinivasan, R., Nicola, V. F., Van Etten, W., and Tattje, H. E. P.: 'Adaptive importance sampling methods for performance evaluation and parameter optimization of communication systems,': IEEE Transactions on Communications, Vol. 48, No. 4, April 2000, pp 557-565.

[4] Srinivasan, R.: 'Simulation of CFAR detection algorithms for arbitrary clutter distributions,' Proc. IEE, Radar, Sonar and Navigation, Part F, Vol. 147, Issue 1, Feb 2000, pp 31-40.

[5] Srinivasan, R.: 'Some results in importance sampling and an application to detection,' Signal Processing, Feb 1998, vol 65, Issue 1, pp 73-88.

[6] Thijs, J., Haartsen, J., Srinivasan, R., and Van Etten, W.: 'Optimizing cell capacity for cellular systems,' IEEE 8th Symposium on Communications and Vehucular Technology in the Benelux, October 18, 2001, Delf, The Netherlands, pp 30-35.

[7] Srinivasan, R.: 'Estimation and approximation of densities of i.i.d. sums via importance sampling,' Signal Processing, Dec 1998, vol 71, Issue 3, pp 235-246. 